\title{
Author Correction: Single-qubit quantum memory exceeding ten-minute coherence time
}

Ye Wang, Mark Um, Junhua Zhang, Shuoming An, Ming Lyu, Jing-Ning Zhang, L.-M. Duan', Dahyun Yum and Kihwan Kim Correction to: Nature Photonics https://doi.org/10.1038/s41566-017-0007-1 (2017); published online 25 September 2017.

In the version of this Letter originally published, in Fig. 2c legend, the entry 'LO phase noise' should not have been included. This has now been corrected in the online versions.

Published online: 12 January 2018

https://doi.org/10.1038/s41566-017-0052-9

\section{Publisher Correction: Giant light-harvesting nanoantenna for single-molecule detection in ambient light}

Kateryna Trofymchuk, Andreas Reisch, Pascal Didier, François Fras, Pierre Gilliot, Yves Mely and Andrey S. Klymchenko Correction to: Nature Photonics https://doi.org/10.1038/s41566-017-0001-7 (2017); published online 29 September 2017.

Owing to a technical error, the wrong version of the Supplementary Information was published for this Article; the equation $E=x_{\text {coupled donors }} \times E_{F R E T}^{\text {local }}$ related to Supplementary Table 6 appeared incorrectly. This error has now been corrected.

\section{Publisher Correction: Chalcogenide glass-on-graphene photonics}

Hongtao Lin, Yi Song, Yizhong Huang, Derek Kita, Skylar Deckoff-Jones, Kaiqi Wang, Lan Li, Junying Li, Hanyu Zheng, Zhengqian Luo, Haozhe Wang, Spencer Novak, Anupama Yadav, Chung-Che Huang, Ren-Jye Shiue, Dirk Englund, Tian Gu, Daniel Hewak, Kathleen Richardson, Jing Kong and Juejun Hu

Correction to: Nature Photonics https://doi.org/10.1038/s41566-017-0033-z (2017); published online 30 October 2017.

In the version of this Article originally published online, the following statement for the equally contributing authors was missing: "Hongtao Lin, Yi Song, Yizhong Huang and Derek Kita contributed equally to this work." This has now been corrected in all versions of the Article. 